\title{
Complementary and alternative treatments of multiple sclerosis: a review of the evidence from 2001 to 2016
}

\author{
Suzi B Claflin, Ingrid A F van der Mei, Bruce V Taylor
}

\begin{abstract}
- Additional material is published online only. To view please visit the journal online (http://dx.doi.org/10.1136/ jnnp-2016-314490)
\end{abstract}

Menzies Institute for Medical Research, University of Tasmania, Hobart, Tasmania, Australia

\section{Correspondence to} Professor Bruce V Taylor, Menzies Institute for Medical Research, University of Tasmania, 17 Liverpool Street, Hobart, TAS 7000, Australia; bruce.taylor@utas.edu.au

Received 9 May 2017

Revised 4 July 2017

Accepted 5 July 2017

Published Online First

2 August 2017

\section{CrossMark}

To cite: Claflin SB, van der Mei IAF, Taylor BV. J Neurol Neurosurg Psychiatry

2018:89:34-41.

\section{ABSTRACT}

People with multiple sclerosis (PwMS) commonly use complementary and alternative medicines (CAM), but an understanding of their efficacy is lacking. Here, we quantitatively review the class I and class II studies of treatment efficacy for multiple sclerosis from January 2001 to January 2017, in order to assess the modern evidence for CAM use. The 38 studies included in this review are divided across five CAM types (cannabis, diet, exercise, psychological approaches and other). We found little evidence to support CAM efficacy. The studies contained little replication in intervention, primary outcomes or study design. Six of 16 CAMs included in this review were only researched in a single study. Future work in this area should build consensus around study methodologies and primary outcomes.

\section{INTRODUCTION}

People with multiple sclerosis (PwMS) commonly use complementary and alternative medicines (CAM) to treat their multiple sclerosis (MS) or associated symptoms. Cross-sectional studies show that $37 \%-100 \%$ of PwMS have ever used CAM, ${ }^{12}$ and up to $51.8 \%$ of PwMS have used CAM in the previous 12 months. ${ }^{3}$ Patients with MS may use CAM in conjunction with pharmacological treatments, and often do not consult either their treating neurologist or general practitioner about their CAM use. ${ }^{1}$ However, PwMS seek advice about CAM use in large numbers, often from alternative sources, especially the internet. Touch Neurology (www.touchneurology.org) reported that non-pharmacological treatments constituted the majority of searches on social media, with diet, exercise, cannabis, vitamin and mineral supplements, and stem cells having the five highest number of searches. Given the high level of interest in CAM, it is essential that medical professionals and PwMS have the best possible understanding of CAM's efficacy and risks.

The interest in CAM treatments within the MS community may result from the perceived and actual shortcomings of available pharmacological treatments. Over the past 20 years, more than 12 immunomodulating treatments for MS have come onto the market. These treatments are highly effective in many cases, but they are not panaceas. MS medications can result in serious side effects and are ineffective for some patients, particularly the $10 \%-15 \%$ of MS cases with primary progressive MS. ${ }^{4}$ Persistent symptoms, perceived lack of effectiveness and adverse reactions to immunomodulating treatments may explain the continued interest of patients with MS in CAM. ${ }^{5}$

CAMs include modifiable factors, like diet and exercise, with their theoretical basis often resting on an accepted, strong relationship between MS incidence or severity and a modifiable risk factor, such as serum vitamin D levels. CAMs often treat by supplementation or cessation, adding the desirable modifiable factor or removing it, as the relationship indicates. Dietary treatments are a popular modifiable CAM for PwMS. ${ }^{25}$ A diverse range of diets, from those high in polyunsaturated fatty acids (PUFA) to gluten free, have been suggested as therapies for MS. ${ }^{6}$ Because the prevalence of MS is greatest in areas with a diet high in saturated fats and low in regions with a diet high in PUFA, it has been suggested that saturated fats might negatively affect patients with MS and that PUFA might be protective or even therapeutic. This has resulted in popular MS-related diets like the Swank diet. Many types of vitamin and herbal supplementation are used by PwMS. Vitamin D is commonly suggested as a therapy, as there are strong associations between latitude, sunlight exposure and MS prevalence and incidence. ${ }^{89}$ It has been inferred that serum vitamin $\mathrm{D}$ concentration moderates these relationships, and this hypothesis has caused speculation that vitamin D supplementation after diagnosis might mitigate MS disease course.

Sun exposure, tobacco cessation and treatment of comorbidities present three other modifiable factor-based CAMs. Sun exposure is a risk factor for central nervous system demyelination independent of the effects of vitamin D discussed above. ${ }^{10}$ There is also a strong association between smoking and MS incidence, as well as more severe disease outcomes. ${ }^{11}$ Similarly, comorbidities have been linked to worse outcomes in MS. ${ }^{12}$ Because of these relationships, tobacco cessation, sun exposure and comorbidity treatment have been suggested as potential therapies.

A diverse range of other CAMs, from acupuncture to bee venom, target the physical and psychological symptoms of MS. Some of the most common are psychological approaches, exercise and cannabis. These include common psychological treatments, such as cognitive-behavioural therapy (CBT), which focuses on the development of coping strategies in order to change unhelpful cognition and behaviour, as well as less conventional techniques like mindfulness. Exercise treatments for both physical and 
psychological symptoms of MS include many different interventions, from yoga to aerobic exercise. ${ }^{2}$ Self-medication with cannabis is a popular symptomatic treatment within the MS community. Cross-sectional surveys of marijuana use have found that between $36 \%$ and $43 \%$ of respondents were ever users of marijuana. ${ }^{13-16}$ The same studies found that the percentage of medicinal users ranged from $15 \%$ to $29.5 \%$.

Thousands of studies have sought to assess the effect of CAM treatments on MS health outcomes. A simple Google search of 'alternative therapies' and 'multiple sclerosis' produces 683000 hits. However, high-quality evidence of efficacy is much harder to find. This scarcity of information is largely due to the difficulty of studying the effects of CAM on MS and the cost of undertaking studies that would unequivocally assess efficacy. While questions like 'Does exercise improve outcomes in MS?' may seem obvious, they are very challenging to answer. The results of such studies are often confounded by reverse causality, small sample sizes, difficult to quantify endpoints and a lack of an appropriate biomarker of improvement. These issues are not unique to CAM studies. They also affect studies of disease-modifying therapies, and have necessitated large multicentre doubleblind randomised clinical trials that cost in the hundreds of millions of dollars to prove effectiveness over a 2 to 3 -year period. ${ }^{17}$

Previous systematic reviews from the Cochrane Collective $^{61819}$ found little evidence of treatment efficacy for dietary, psychological or exercise interventions for MS in the literature from 1966 to the 2000s. We recommend these as comprehensive systematic reviews. Building on this work, we reviewed CAM studies published from 2001 onwards, presenting class I and class II evidence of efficacy and summarising their findings. We will broadly assess the most recent evidence of CAM efficacy, identify common potential sources of bias and suggest methodological improvements for further development of the field. Our goal is to provide an overview of the modern evidence for CAM use, and highlight future directions for this important area of research.

\section{METHODS}

The WHO has defined CAM as 'a broad set of health care practices that are not part of that country's own tradition and are not integrated into the dominant health care system.' For the purposes of this review, we have broadened the WHO definition of CAM to a non-invasive therapy used in addition to or in lieu of the standard pharmacological treatment of MS. We focused on papers available through the PubMed database. Online supplementary appendix 1 presents the search terms.

The final search was conducted on 18 January 2017, returning 1916 entries. The entries were evaluated in three sequential steps.

First, by the following inclusion criteria:

1. Must be a full research article-this review did not include study protocols or abstracts.

2. Must be published in the period between 1 January 2001 and 18 January 2017, as 2001 was the first year that the McDonald criteria for MS diagnosis were published, which may improve uniformity in MS diagnosis.

3. Must be in English.

4. Must have only human participants.

5. Must test the effect of a CAM treatment on health outcomes in patients with MS only.

Second, we evaluated by sample size, requiring a minimum of 50 total participants (including both treatment and control groups) in the analysis. This criterion aimed to eliminate studies that had an insufficient sample size and thus are at high risk of type II error.

Third, we evaluated by class of evidence, including studies that presented class I or class II evidence of efficacy. Class of evidence was assessed using a modified version of Evidence-Based SpineCare Journal (2013)'s standard ${ }^{20}$ (see online supplementary appendix 2), which was selected for its clarity. Studies were evaluated against seven major criteria. Random sequence generation required random treatment assignment. Allocation concealment required a convincing placebo that obscured treatment assignment to participants. Blind or independent assessment was evaluated based on stated blinding procedures or protocols. Equal application of cointerventions was assessed based on the size of treatment groups at randomisation. Follow-up rate was determined by comparing the group size included in analysis with the randomised group. The criteria for adequate sample size required the demonstration of a power calculation and a sample size that met the suggested sample size from that calculation. Intention-to-treat analysis was evaluated by reviewing the methods section and the tables and figures to see if this approach was indicated.

Because the assessment for each criterion was strict, the cut-off for class I evidence was rigorous. In order to be considered class I, a study had to fulfil all seven criteria. In order to ensure that we captured all high-quality studies and were not overly harsh, we were generous with the distinction between class II and III evidence. Class II studies could violate up to three of the criteria for class I evidence. A study had to violate four or more criteria to be designated class III.

The citations of included articles, as well as articles that met all inclusion criteria except class of evidence, were reviewed for other studies of interest, adding 11 studies, which were then evaluated against the inclusion criteria. One paper $^{21}$ was included based on personal knowledge and was assessed by the same criteria. A single author (SBC) reviewed all articles.

Of the 1475 articles remaining after the initial language and date filtering and the addition of the 12 articles from citations and personal knowledge, 1176 were excluded based on study objective or design. A further 191 were excluded based on sample size. Finally, 52 articles were excluded based on class of evidence. Figure 1 depicts the process.

For the purposes of this review, a study was defined by its research cohort. Articles that assessed different subsets of a larger cohort were considered distinct studies. Altogether, 38 distinct studies met the inclusion criteria. Included studies were categorised by the type of CAM treatment they researched: cannabis, diet, exercise, psychological approaches and other (table 1). Studies were compared within groups for study design, results and class of evidence. When multiple articles from the same study were reviewed, we refer to the study by the citation of the article published first. (Online supplementary appendix 3 lists all included articles.) The data from each study were extracted by one researcher and entered into a standardised data collection sheet.

\section{RESULTS}

Nearly half $(48.1 \%)$ of the articles that met all other inclusion criteria were excluded based on class of evidence, leaving 38 randomised controlled trial (RCT) studies included in this review. Four of these present class I evidence, three studying cannabis ${ }^{22-24}$ and one studying Ginkgo biloba supplementation. ${ }^{25}$ The remaining 34 studies present class II evidence (table 1). Table 2 contains summary statistics for the class of evidence assessment, showing the percentage of studies fulfilling each criterion. 


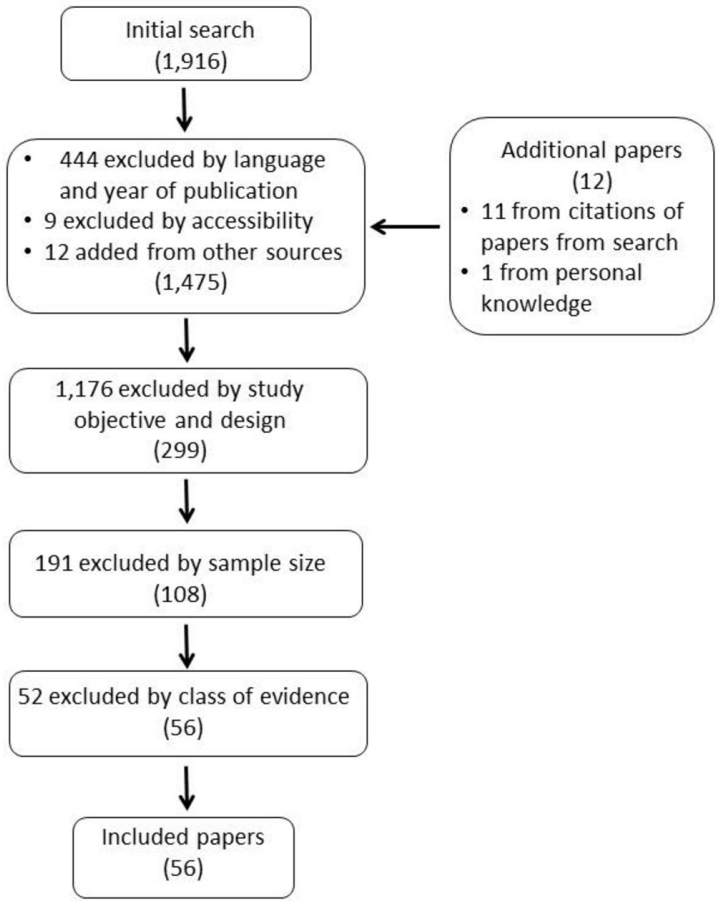

Figure 1 Inclusion flow chart depicting the process of evaluation for this review.

The most common sources of bias were a lack of intention-totreat analysis, no allocation concealment and inadequate sample size. Generally, the studies that could conceal treatment allocation did so. However, allocation concealment was not possible for the exercise, psychological and all but one of the 'other' studies, because the treatment could not be obscured. Although this is unavoidable, unblinded participants still present a potential source of bias for these studies via the placebo effect and possible reverse causality. The most common avoidable source of bias was sample size, where a study did not present a power calculation of any kind and/or did not include a sample size that fulfilled the requirements of that power calculation. Sample sizes that do not fulfil power calculations should also be justified for the sake of transparency. Inadequate sample size is a significant potential source of bias, leaving these studies vulnerable to type II error.

All CAM categories included a wide range of primary outcomes (see online supplementary appendix 4). Even after grouping outcomes into overarching categories, few outcomes were assessed in more than $50 \%$ of studies in a particular group. Instead, there was little overlap between studies, and therefore little of the replication necessary to draw conclusions about treatment efficacy.
Cumulatively, we reviewed 16 different interventions. Table 3 presents this information in an abbreviated format. All articles are summarised in online supplementary appendix 5 .

\section{Cannabis}

Seven studies on the effect of cannabis met the inclusion criteria. Five studies found that cannabis extract, including Sativex, had a significant beneficial effect on MS health outcomes. Three double-blind RCTs with sample sizes ranging from $64^{22}$ to $224^{23}$ and $225^{24}$ participants presented class I evidence. These studies demonstrate that cannabis extract can significantly improve incontinence, ${ }^{24}$ pain ${ }^{22}$ and spasticity ${ }^{23}$ compared with placebo. Class II evidence from two double-blind RCTs demonstrates a significant improvement in spasticity ${ }^{26}$ and patient-reported muscle stiffness ${ }^{27}$ compared with placebo. These studies are high quality, with sample sizes of 189 and 279 participants analysed, respectively.

Two other high-quality studies found no effect of cannabis extract on primary outcomes. Wade et al found no effect on the visual analogue score of the individual's most troublesome symptom $(\mathrm{n}=154) .{ }^{28}$ Zajicek et al found no effect on spasticity $(\mathrm{n}=611){ }^{29}$

Two studies assessed the impact of tetrahydrocannabinol (THC) extract on MS. One study presented class I evidence demonstrating that orally administered THC improved the adjusted incontinence episode rate by $33 \%$, significantly more than placebo. ${ }^{24}$ Conversely, the results of one class II doubleblind RCT found no effect on spasticity. ${ }^{29}$

Four of the reviewed studies assessed safety and/or tolerability of cannabis extract or THC treatment, ${ }^{22} 262829$ and all found limited adverse effects.

\section{Diet}

Seven studies on the effect of diet were included in this review. Vitamin D supplementation was the most common intervention, with three studies. In a 96-week double-blind class II study analysing 68 fully ambulatory people with relapsing-remitting multiple sclerosis (RRMS), Steffensen et $a l^{30}$ found that vitamin $\mathrm{D}_{3}$ supplementation with $20000 \mathrm{IU} /$ week had no effect on annualised relapse rate, disability (Expanded Disability Status Scale; EDSS), multiple sclerosis functional composite components, grip strength, fatigue, inflammation markers or bone loss compared with a calcium stablet control. In a double-blind RCT analysing 59 participants, Mosayebi et $a l^{31}$ also found that short-term vitamin D therapy had no effect on disability or gadolinium-enhancing lesions after 6 months. However, at the 6-month follow-up, vitamin D supplementation resulted in significantly lower cell proliferation, and a $79.3 \%$ higher transforming growth factor-beta concentration and $37.5 \%$ higher interleukin-10 concentration.

\begin{tabular}{lcllll}
\hline \multicolumn{2}{l}{ Table 1} & Summary statistics of included studies & & & \\
\hline CAM & Number of studies & Percentage of studies & Class I & Class II & Average number of participants \\
\hline Cannabis & 7 & 18.4 & 3 & 4 & 249.4 \\
Diet & 7 & 18.4 & 1 & 6 & 88 \\
Exercise & 10 & 26.3 & 0 & 10 & 92.4 \\
Psychological approaches & 9 & 13.2 & 0 & 9 & 106 \\
Other & 5 & & 0 & 5 & 72.2 \\
Total & 38 & 4 & 34 & 121.6 \\
\hline
\end{tabular}

CAM, complementary alternative medicines. 
Table 2 Summary statistics for class of evidence assessment of studies included in this review

\begin{tabular}{|c|c|c|c|c|c|c|}
\hline CAM type & Cannabis $(n=7)$ & Diet $(n=7)$ & Exercise $(n=10)$ & $\begin{array}{l}\text { Psychological } \\
\text { approaches }(n=9)\end{array}$ & Other $(n=5)$ & Total $(n=38)$ \\
\hline Adequate sample size & 4 & 3 & 3 & 3 & 3 & $16(42.1 \%)$ \\
\hline Follow-up rate of $80 \%+$ & 7 & 6 & 10 & 8 & 4 & $37(97.4 \%)$ \\
\hline Random sequence generation & 7 & 7 & 10 & 8 & 5 & $38(100.0 \%)$ \\
\hline Allocation concealment & 7 & 7 & 0 & 0 & 2 & $15(39.5 \%)$ \\
\hline Blind assessment of main outcomes & 7 & 7 & 9 & 5 & 3 & $33(86.8 \%)$ \\
\hline Equal intervention application & 6 & 6 & 10 & 8 & 4 & $36(94.7 \%)$ \\
\hline Intent-to-treat analysis & 4 & 3 & 6 & 7 & 0 & $20(52.6 \%)$ \\
\hline
\end{tabular}

CAM, complementary alternative medicines.

The remaining study suggests that vitamin D supplementation may be beneficial as an add-on treatment. In a double-blind RCT analysing 62 PwMS, Soilu-Hänninen $e a^{l^{32}}$ demonstrated that as an add-on treatment to interferon-beta, vitamin $\mathrm{D}_{3}$ significantly reduced the number of T1 gadolinium-enhancing MRI lesions by $83.3 \%$. They also found that it was well tolerated. However, it had no effect on the primary outcomes, burden of disease and disability accumulation. Collectively, these studies demonstrate that vitamin D had little effect on MS health outcomes.

Two class II double-blind studies assessed the impact of PUFA supplementation. The first study, Rezapour-Firouzi et al, ${ }^{33}$ also tested the impact of a hot-natured diet, a concept derived from traditional Iranian medicine, which attributes a 'heating' or 'cooling' effect to different foods, as well as the effect of PUFA-rich oils. A hot-natured diet is associated with low MS prevalence, and is thought to enhance T-helper (Th2) immune responses, based on preliminary research. ${ }^{34}$ The authors analysed 65 patients with RRMS with an EDSS <6.0. They found that PUFA-rich oils, both with and without a hot-natured diet intervention, decreased relapse rate, and decreased disability (EDSS) over 6 months. However, although the olive oil control did not result in significant reductions of the primary outcomes, the authors did not statistically compare the treatment and control groups, making it difficult to assess treatment effects. Additionally, the conclusions of this study must be interpreted cautiously, as it had a small sample size (65 participants across three treatments) and a high loss to follow-up (35\%).

The second and more rigorous study by Torkildsen et $a l^{35}$ evaluated the effect of omega-3 supplementation in a 4-year trial. This study included 92 PwMS, divided between the treatment and control groups, and had greater than $80 \%$ follow-up. The authors found no effect of omega-3 intake on gadolinium-enhancing MRI lesions or on the tertiary outcome of disability.

Two dietary interventions were assessed in only one study: biotin and G. biloba (Ginkgo). Tourbah et al, a double-blind RCT analysing 154 participants, ${ }^{21}$ found that high-dose biotin (MD1003) reduced disability progression and improved the clinical impression of change in patients with progressive MS compared with placebo. It also resulted in a sustained reversal of disability in $12.6 \%$ of treated patients, which was significantly more than the control (0 patient). Lovera et al, ${ }^{25}$ a class I double-blind RCT analysing 116 participants, found that Ginkgo extract does not have a significant effect on cognitive function compared with a placebo, but is well tolerated.

\section{Exercise}

Exercise had the greatest representation in this review, with 10 included studies. Seven studies researched the effect of exercise or physical activity generally. Of these, three studies assessed the effect of individual exercise interventions compared with no intervention controls. The first, an unblinded RCT of 91 patients with MS with mild to moderate disability, ${ }^{36}$ found that a 6 -month exercise programme increased walking speed significantly more than the control, but had no effect on health-related quality of life (HR-QoL). The second, a single-blinded RCT of 71 PwMS, ${ }^{37}$ showed that the effects of exercise may be time sensitive. The authors found that while physical activity significantly improved the secondary outcomes of muscle endurance and strength, psychological assessment and mobility at 10 weeks, these effects were not sustained at a long-term follow-up ( 22 weeks). The third study found no effect of aerobic exercise on attentiveness or alertness compared with a waitlist control $(n=57) .^{38}$

Two studies, both single-blinded RCT, looked at the impact of group exercise interventions compared with no intervention controls. They found that group exercise training significantly improves primary and secondary outcomes, including balance, ${ }^{39}$ walking speed, ${ }^{39}{ }^{40}$ fatigue $^{39}$ and MS Impact Scale (MSIS) physical and psychological components. ${ }^{40}$

Two single-blinded RCTs compared different exercise interventions. Collett et $a l^{41}$ found that a 6 -week exercise programme improved mobility from baseline (effect size $=0.25$ ). However, they determined that the intensity (continuous, intermittent or combined) of exercise did not significantly affect the change. Bansi et $a l^{42}$ found that there were no differences in the cytokine and neurotrophin concentrations between treatments: endurance training on a cycle ergometer or on an aquatic bike.

Three studies explored the impact of different innovative balance and gait training approaches. Two studies demonstrated that sensory integration balance training ${ }^{43}$ and robot-assisted gait training ${ }^{44}$ significantly improve the primary outcomes of balance ${ }^{43} 44$ and walking endurance, ${ }^{44}$ and the secondary outcome of fatigue ${ }^{43}$ compared with conventional rehabilitation control. However, Nilsagård et al found no effect of Nintendo Wii Fit balance training on patient balance compared with a non-exercise control group. ${ }^{45}$

Two studies assessed the effects of yoga. The first, a singleblind RCT analysing 57 PwMS, found that yoga had no effect on attention and alertness compared with a waitlist control. ${ }^{38}$ The second, a single-blind RCT analysing 291 PwMS, ${ }^{40}$ found that group yoga did not affect the primary outcome of the MSIS physical component or the secondary outcome of walking speed. It did improve other secondary outcomes, such as Modified Fatigue Impact Scale total score and its psychological component.

\section{Psychological approaches}

Psychological approaches for treating MS symptoms were assessed in nine studies. The most commonly studied psychological CAM was CBT. Seven class II RCTs studying the effect 


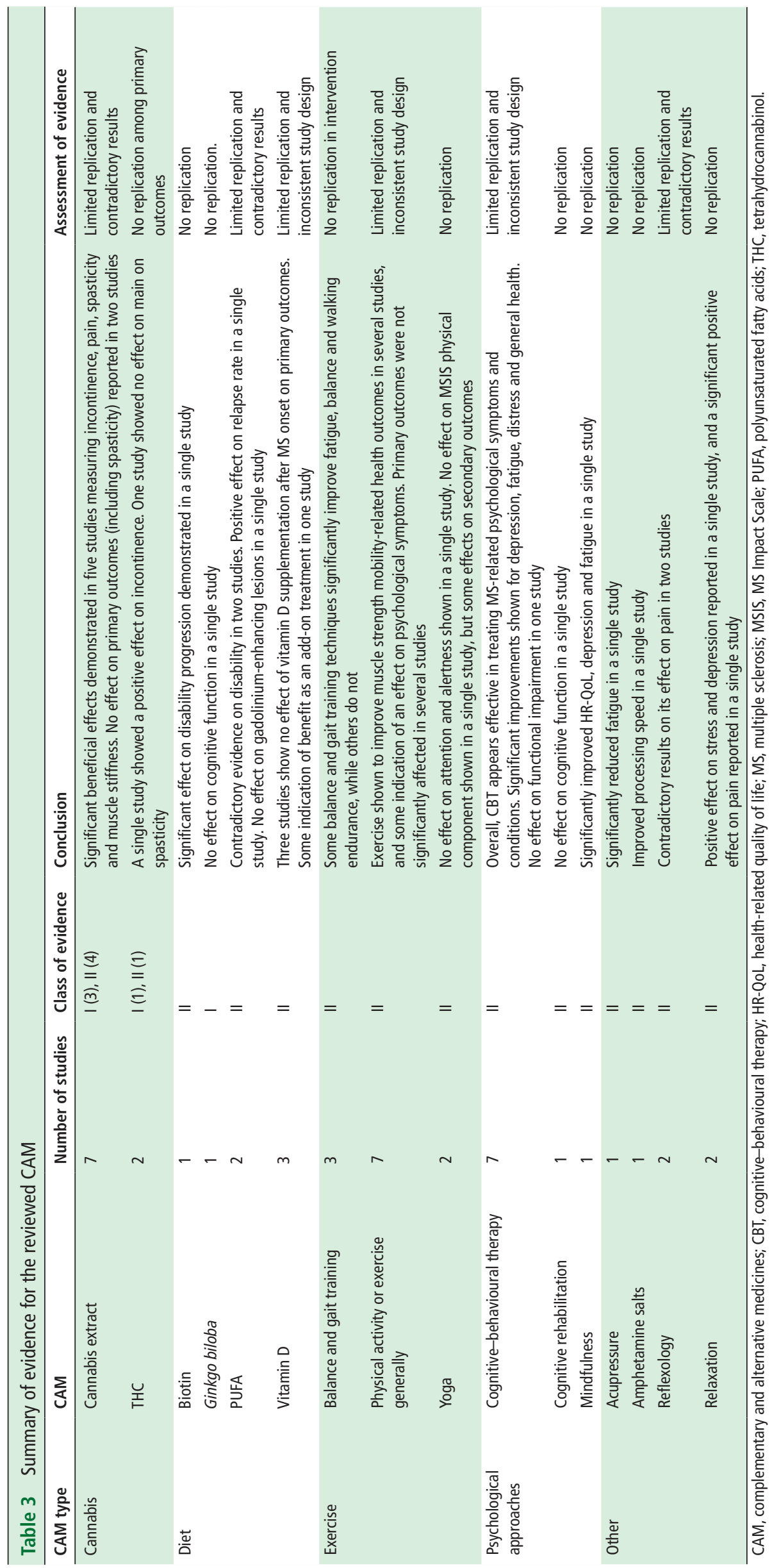


of CBT were included. However, these studies used a range of methodologies, making them difficult to compare directly.

One study evaluated the impact of CBT compared with a no intervention control. This single-blind study ${ }^{46}$ found that CBT significantly improved general health score (General Health Questionnaire-12) by $5.5 \%$, as well as the secondary outcomes of anxiety and depression. CBT also performed well in the six class II RCTs where it was compared with other interventions. Compared with supportive emotion-focused therapy, CBT improved quality of life, ${ }^{47}$ fatigue ${ }^{47}$ and disability. ${ }^{48}$ Moss-Morris et $a l^{49}$ found that, when compared with supportive listening, CBT significantly decreased patient distress score by $19.2 \%$ (General Health Questionnaire-12) 1 year after treatment, but had no effect on functional impairment. In the same study, the authors found that CBT was especially effective for those with little social support and high levels of distress. van Kessel et al $l^{50}$ found that CBT was significantly more effective than relaxation therapy as a treatment for fatigue, although both were clinically effective (effect sizes of 3.03 and 1.83, respectively). An assessor-blind RCT of 146 PwMS found that a CBT-based group MS-fatigue management programme significantly improved fatigue severity and self-efficacy compared with current local practice alone, and that these effects persisted 1 year after the intervention with effect sizes of -0.29 and 0.34 , respectively. ${ }^{51}$ Similarly, Mohr et $a l^{52}$ found that CBT and the antidepressant sertraline were significantly more effective than supportive-expressive group therapy at treating depression, although all three were clinically effective. The authors also found that reductions in depression were associated with reductions in fatigue, measured with the Fatigue Assessment Instrument.

Two psychological approaches were assessed in a single study: mindfulness and cognitive rehabilitation. Grossman et al, an unblinded class II RCT, ${ }^{53}$ found that a mindfulness-based intervention improved HR-QoL, quantified with both a disease-aspecific and a disease-specific measure (effect sizes of 0.51 and 0.28 , respectively). The intervention also improved fatigue (effect size of 0.38) and depression (effect size of 0.356). The treatment had significantly better outcomes than the usual care control. Stuifbergen et al, ${ }^{54}$ a single-blind RCT of 61 PwMS, found that a computer-assisted cognitive rehabilitation intervention was not significantly different in improving most measures on the Minimal Assessment of Cognitive Function in MS battery compared with a waitlist control, but was associated with greater improvement in compensatory strategies.

\section{Other CAMs}

There is a wide range of CAMs available to patients with MS, many of which defy simple classification schemes. In this section, we will summarise the results of studies that met our inclusion criteria, but were difficult to sort into a larger group of studies. This group of five studies encompassed a variety of treatment types, ranging from amphetamine salts to acupressure. All studies were RCT presenting class II evidence.

Two studies of relaxation techniques met inclusion criteria. In an assessor-blind study of 61 people with RRMS, Artemiadis et $a l^{55}$ found that a stress management technique combining relaxation breathing and progressive muscle relaxation exercises significantly reduced patient-reported stress (Perceived Stress Scale, effect size $=0.62$ ) and depression (Beck Depression Inventory, effect size $=0.53$ ) compared with no intervention. In an assessor-blind study of 75 female patients with MS, Nazari et $a l^{56}$ found that relaxation significantly improved participant pain compared with no intervention. However, this effect was immediate and did not continue at the 2-month follow-up, indicating that it may have been a placebo effect.

Two studies of reflexology, the specific application of pressure to the feet and hands, met the inclusion criteria, and present contradictory results. The first, a single-blind RCT analysing 75 female patients with MS, found that reflexology significantly improved pain intensity (Numerical Rating Scale) compared with both relaxation and no intervention controls immediately post-treatment. ${ }^{56}$ However, this effect was no longer present at a 2-month follow-up, indicating that it may have been a placebo effect. The second study, a single-blind RCT analysing 73 PwMS,${ }^{57}$ found that reflexology did not significantly affect pain (Visual Analogue Scale) compared with a sham control, although both caused a significant decrease that persisted at the 12-week follow-up.

Two CAMs in this category were assessed in one study: acupressure and amphetamine salts. An unblinded $\mathrm{RCT}^{58}$ found that acupressure, which stimulates acupoints but does not use needles, significantly reduced fatigue compared with placebo. The authors found that acupressure reduced fatigue score (Fatigue Severity Scale) by $26 \%$ at a 4 -week follow-up. In a double-blind RCT analysing 52 patients with MS with processing impairment, Morrow and Rosehart ${ }^{59}$ found that mixed amphetamine salts extended release significantly improved processing speed compared with placebo.

\section{DISCUSSION}

Overall, we found little evidence of efficacy for CAM treatments of MS and class I evidence was almost universally lacking. As outlined above, there are dozens of studies showing a positive effect of a particular CAM on a particular health outcome, but there is little overlap or consistency between studies, making it impossible to draw meaningful conclusions about treatment efficacy. Six of the 16 CAMs included in this review were each assessed in a single study, offering no replication. The CAMs with the strongest evidence supporting them, cannabis extract, physical activity and CBT, were each investigated in seven studies. Yet, these studies had different designs and methodologies, and in some cases contradictory results. These issues make the results difficult to synthesise into an assessment of treatment efficacy, and thus into recommendations for translation and implementation. This is not to say that CAMs have no effect on MS, but that there is currently no rigorous scientific evidence to support their use. In large part, the absence of evidence reflects the difficulties in undertaking high-quality efficacy studies.

Our findings are largely in agreement with several previous reviews on the subject, ${ }^{6061}$ as well as National Institute for Health and Care Excellence guidelines for the management of MS. ${ }^{62}$ A recent review by the American Academy of Neurology ${ }^{63}$ did provide some guidelines on CAM efficacy in MS, but used relatively lenient criteria for evidence of effectiveness.

Farinotti et $a l,{ }^{6}$ who systematically reviewed dietary interventions for MS from 1966 to 2006, included only six studies in their analysis and found no evidence that dietary interventions affected relapse or disease progression, as measured by disability. Our review comes to a similar conclusion. We found a lack of any replicated evidence that diet affects MS outcomes that cannot be attributed to type I or type II errors, placebo effect or reverse causality.

The same issues impact most other CAMs included in this review. The studies reviewed here show that exercise can improve health outcomes for patients with MS, particularly those related to mobility and muscular strength, but there is little replication in 
the data and inconsistency in study design. This agrees with Rietberg et al's review of the subject. They systematically reviewed the literature from 1966 to 2004, and found only nine studies of exercise therapy for MS that met their inclusion criteria for highquality studies. While they found strong evidence that exercise therapy improves muscle power, mobility and exercise tolerance functions, the authors point out that much of this evidence was derived from studies with small sample sizes that are vulnerable to type II error. The nine studies included in their review cumulatively included 260 participants. $^{19}$

Generally, the studies in this review present good evidence that CBT is an effective treatment of psychological symptoms of MS and conditions related to MS, including depression and distress. There is also some evidence that it affects fatigue and general health scores. However, as with exercise, there is significant variability in study methodologies and in outcomes of interest. Thomas et $a l^{18}$ reached a similar conclusion in their systematic review of the literature from 1966 to 2004. The authors included 16 studies and, while they stated there was reasonable evidence that cognitive-behavioural approaches were effective on MS-related depression, they found that due to the wide range of interventions, study designs and outcomes of interest, they could not conclude anything about treatment efficacy.

\section{Methodological improvements to further develop the field} Greater consistency in study methodology and design is the most important methodological improvement needed for CAMs research related to MS. The current breadth of studies in this field is both advantageous and disadvantageous to our understanding of CAM's impact. The range of these studies gives some information on many topics, and begins the work of exploring the effect of CAM treatment. On the other hand, it presents limited to no replication of findings. As discussed above, without the depth of knowledge garnered from repeated research, few conclusions can be reached.

In order to improve our understanding of the effects of CAM on MS health outcomes, future work should cultivate methodological consistency by establishing standard control or comparator groups and outcome measures. Currently, studies that assess the same CAM are difficult to evaluate together because their results reflect different comparisons. The field would also be well served by establishing outcomes of interest, which would allow for greater replication and the accrual of a depth of evidence about an outcome.

We would strongly advocate for the establishment of a CAM trial group within the MS research community. This would allow for the development of MS-specific trial structures for CAM studies. CAM treatment is clearly an area of significant interest to the MS community and should be a research priority.

\section{Future research directions}

There is a great deal of work left to be done in this area of research. While any high-quality study in this field stands to make a significant contribution, we will put forward four CAMs from this review and three that are absent as especially interesting for future work.

The three best supported CAMs in this review, cannabis extract, physical activity and CBT, should be further studied in order to better understand their effectiveness as MS treatments. They merit particular focus because a positive effect has already been demonstrated by several high-quality studies, indicating that these may be fruitful lines of research. Additionally, the effect of biotin should be explored in more depth. Although only one study in this review assessed the impact of biotin, it showed a positive effect on disability in patients with progressive-type MS. Because of the limited treatment options for progressive MS, biotin is particularly deserving of future study. We await with interest the results of the phase III biotin trial, further vitamin D studies and other dietary RCTs in early and established MS that are currently under way around the world.

Many of the CAMs used to treat MS were not included in this review (see online supplementary appendix 6 for a list of the most common). We feel that three of these are of particular interest: tobacco cessation, sunlight exposure and comorbidity treatment. All of these areas have strong theoretical underpinnings that support their hypothesised effects. They have the additional benefit of being modifiable factors that can be implemented by PwMS relatively easily. In the case of sunlight exposure and tobacco cessation, they also pose little to no risk of adverse events, making them particularly attractive as potential treatments.

There is a great deal of interest in CAM treatment options within the MS community. Unfortunately, efficacy studies of CAM treatments present significant challenges, and as this review demonstrates, there is little class I and class II evidence of their effect in the literature. The impact of CAM treatments should be assessed more rigorously in future research.

Contributors SBC completed the review and literature review under the supervision of BVT. IAFvdM assisted with all apsects of critical review of the manuscript.

\section{Competing interests None declared.}

Provenance and peer review Commissioned; externally peer reviewed.

(C) Article author(s) (or their employer(s) unless otherwise stated in the text of the article) 2018. All rights reserved. No commercial use is permitted unless otherwise expressly granted.

\section{REFERENCES}

1 Pucci E, Cartechini E, Taus C, et al. Why physicians need to look more closely at the use of complementary and alternative medicine by multiple sclerosis patients. Eur J Neurol 2004;11:263-7.

2 Stoll SS, Nieves C, Tabby DS, et al. Use of therapies other than disease-modifying agents, including complementary and alternative medicine, by patients with multiple sclerosis: a survey study. J Am Osteopath Assoc 2012;112:22-8.

3 Skovgaard L, Nicolajsen PH, Pedersen E, et al. Differences between users and non-users of complementary and alternative medicine among people with multiple sclerosis in Denmark: a comparison of descriptive characteristics. Scand J Public Health 2013;41:492-9.

4 Koch $\mathrm{M}$, Kingwell $\mathrm{E}$, Rieckmann $\mathrm{P}$, et al. The natural history of primary progressive multiple sclerosis. Neurology 2009;73:1996-2002.

5 Schwarz S, Knorr C, Geiger H, et al. Complementary and alternative medicine for multiple sclerosis. Mult Scler 2008;14:1113-9.

6 Farinotti M, Simi S, Di Pietrantonj C, et al. Dietary interventions for multiple sclerosis. Cochrane Database Syst Rev 2007:CD004192.

7 Swank RL, Dugan BB. Effect of low saturated fat diet in early and late cases of multiple sclerosis. Lancet 1990;336:37-9.

8 Simpson S, Blizzard L, Otahal P, et al. Latitude is significantly associated with the prevalence of multiple sclerosis: a meta-analysis. J Neurol Neurosurg Psychiatry 2011;82:1132-41

9 Alonso A, Hernán MA. Temporal trends in the incidence of multiple sclerosis: a systematic review. Neurology 2008;71:129-35

10 Lucas RM, Ponsonby AL, Dear K, et al. Sun exposure and vitamin D are independent risk factors for CNS demyelination. Neurology 2011;76:540-8.

11 Pittas F, Ponsonby AL, van der Mei IA, et al. Smoking is associated with progressive disease course and increased progression in clinical disability in a prospective cohort of people with multiple sclerosis. J Neurol 2009;256:577-85.

12 Tettey P, Siejka D, Simpson S, et al. Frequency of Comorbidities and their association with clinical disability and relapse in multiple sclerosis. Neuroepidemiology 2016:46:106-13

13 Chong MS, Wolff $\mathrm{K}$, Wise $\mathrm{K}$, et al. Cannabis use in patients with multiple sclerosis. Mult Scler 2006:12:646-51.

14 Clark AJ, Ware MA, Yazer E, et al. Patterns of Cannabis use among patients with multiple sclerosis. Neurology 2004;62:2098-100 
15 Page SA, Verhoef MJ, Stebbins RA, et al. Cannabis use as described by people with multiple sclerosis. Can J Neurol Sci 2003;30:201-5.

16 Linassi AG, Hader WJ. Perceived effects of Marijuana use by MS patients in Saskatchewan-a pilot study. Int J MS Care 2003;5:139-50.

17 Lublin F, Miller DH, Freedman MS, et al. Oral fingolimod in primary progressive multiple sclerosis (INFORMS): a phase 3, randomised, double-blind, placebocontrolled trial. Lancet 2016;387:1075-84.

18 Thomas PW, Thomas S, Hillier C, et al. Psychological interventions for multiple sclerosis (Review). Cochrane Database Syst Rev 2006;1:CD004431.

19 Rietberg MB, Brooks D, Uitdehaag BMJ, et al. Exercise therapy for multiple sclerosis (Review). Cochrane Database Syst Rev 2004;1:CD003980.

20 Definition of the different classes of evidence (CoE). Evid Based Spine Care J 2013:4:167.

21 Tourbah A, Lebrun-Frenay C, Edan G, et al. MD1003 (high-dose biotin) for the treatment of progressive multiple sclerosis: a randomised, double-blind, placebocontrolled study. Mult Scler 2016;22:1719-31.

22 Rog DJ, Nurmikko TJ, Friede T, et al. Randomized, controlled trial of cannabis-based medicine in central pain in multiple sclerosis. Neurology 2005;65:812-9.

23 Novotna A, Mares J, Ratcliffe S, et al. A randomized, double-blind, placebo-controlled, parallel-group, enriched-design study of nabiximols* (Sativex $(\circledR))$, as add-on therapy, in subjects with refractory spasticity caused by multiple sclerosis. Eur J Neurol 2011:18:1122-31.

24 Freeman RM, Adekanmi O, Waterfield MR, et al. The effect of Cannabis on urge incontinence in patients with multiple sclerosis: a multicentre, randomised placebocontrolled trial (CAMS-LUTS). Int Urogynecol J 2006;17:636-41.

25 Lovera JF, Kim E, Heriza E, et al. Ginkgo biloba does not improve cognitive function in MS: a randomized placebo-controlled trial. Neurology 2012;79:1278-84.

26 Collin C, Davies P, Mutiboko IK, et al. Randomized controlled trial of cannabis-based medicine in spasticity caused by multiple sclerosis. Eur J Neurol 2007;14:290-6.

27 Zajicek JP, Hobart JC, Slade A, et al. Multiple sclerosis and extract of Cannabis: results of the MUSEC trial. J Neurol Neurosurg Psychiatry 2012;83:1125-32.

28 Wade DT, Makela P, Robson P, et al. Do cannabis-based medicinal extracts have general or specific effects on symptoms in multiple sclerosis? A double-blind, randomized, placebo-controlled study on 160 patients. Mult Scler 2004;10:434-41.

29 Zajicek J, Fox P, Sanders H, et al. Cannabinoids for treatment of spasticity and other symptoms related to multiple sclerosis (CAMS study): multicentre randomised placebo-controlled trial. Lancet 2003;362:1517-26.

30 Steffensen LH, Jørgensen L, Straume B, et al. Can vitamin D supplementation prevent bone loss in persons with MS? A placebo-controlled trial. J Neurol 2011;258:1624-31.

31 Mosayebi G, Ghazavi A, Ghasami K, et al. Therapeutic effect of vitamin D3 in multiple sclerosis patients. Immunol Invest 2011;40:627-39.

32 Soilu-Hänninen M, Aivo J, Lindström BM, et al. A randomised, double blind, placebo controlled trial with vitamin D3 as an add on treatment to interferon $\beta$-1b in patients with multiple sclerosis. J Neurol Neurosurg Psychiatry 2012;83:565-71.

33 Rezapour-Firouzi S, Arefhosseini SR, Mehdi F, et al. Immunomodulatory and therapeutic effects of Hot-nature diet and co-supplemented hemp seed, evening primrose oils intervention in multiple sclerosis patients. Complement Ther Med 2013;21:473-80.

34 Shahabi S, Hassan ZM, Mahdavi M, et al. Hot and Cold natures and some parameters of neuroendocrine and immune systems in traditional iranian medicine: a preliminary study. J Altern Complement Med 2008;14:147-56.

35 Torkildsen Øivind, Wergeland S, Bakke $\mathrm{S}$, et al. $\omega$-3 Fatty acid treatment in multiple sclerosis (OFAMS study). Arch Neurol 2012;69:1044-51.

36 Romberg A, Virtanen A, Ruutiainen J, et al. Effects of a 6-month exercise program on patients with multiple sclerosis: a randomized study. Neurology 2004;63:2034-8.

37 Dodd KJ, Taylor NF, Shields N, et al. Progressive resistance training did not improve walking but can improve muscle performance, quality of life and fatigue in adults with multiple sclerosis: a randomized controlled trial. Mult Scler 2011;17:1362-74

38 Oken BS, Kishiyama S, Zajdel D, et al. Randomized controlled trial of yoga and exercise in multiple sclerosis. Neurology 2004;62:2058-64.

39 Tarakci E, Yeldan I, Huseyinsinoglu BE, et al. Group exercise training for balance, functional status, spasticity, fatigue and quality of life in multiple sclerosis: a randomized controlled trial. Clin Rehabil 2013:27:813-22.

40 Garrett M, Hogan N, Larkin A, et al. Exercise in the community for people with minimal gait impairment due to MS: an assessor-blind randomized controlled trial. Mult Scler 2013;19:782-9.
41 Collett J, Dawes H, Meaney A, et al. Exercise for multiple sclerosis: a single-blind randomized trial comparing three exercise intensities. Mult Scler 2011;17:594-603.

42 Bansi J, Bloch W, Gamper U, et al. Training in MS: influence of two different endurance training protocols (aquatic versus overland) on cytokine and neurotrophin concentrations during three week randomized controlled trial. Mult Scler 2013;19:613-21.

43 Gandolfi M, Munari D, Geroin C, et al. Sensory integration balance training in patients with multiple sclerosis: a randomized, controlled trial. Mult Scler 2015;21:1453-62.

44 Straudi S, Fanciullacci C, Martinuzzi C, et al. The effects of robot-assisted gait training in progressive multiple sclerosis: a randomized controlled trial. Mult Scler 2016:22:373-84.

45 Nilsagård YE, Forsberg AS, von Koch L. Balance exercise for persons with multiple sclerosis using Wii games: a randomised, controlled multi-centre study. Mult Scler 2013;19:209-16.

46 Lincoln NB, Yuill F, Holmes J, et al. Evaluation of an adjustment group for people with multiple sclerosis and low mood: a randomized controlled trial. Mult Scler 2011:17:1250-7.

47 Mohr DC, Hart SL, Julian L, et al. Telephone-administered psychotherapy for depression. Arch Gen Psychiatry 2005:62:1007-14.

48 Mohr DC, Hart S, Vella L. Reduction in disability in a randomized controlled trial of telephone-administered cognitive-behavioral therapy. Health Psychol 2007:26:554-63.

49 Moss-Morris R, Dennison L, Landau S, et al. A randomized controlled trial of cognitive behavioral therapy (CBT) for adjusting to multiple sclerosis (the saMS trial): does CBT work and for whom does it work? J Consult Clin Psychol 2013:81:251-62.

50 van Kessel K, Moss-Morris R, Willoughby E, et al. A randomized controlled trial of cognitive behavior therapy for multiple sclerosis fatigue. Psychosom Med 2008;70:205-13

51 Thomas S, Thomas PW, Kersten P, et al. A pragmatic parallel arm multi-centre randomised controlled trial to assess the effectiveness and cost-effectiveness of a group-based fatigue management programme (FACETS) for people with multiple sclerosis. J Neurol Neurosurg Psychiatry 2013;84:1092-9.

52 Mohr DC, Boudewyn AC, Goodkin DE, et al. Comparative outcomes for individual cognitive-behavior therapy, supportive-expressive group psychotherapy, and sertraline for the treatment of depression in multiple sclerosis. J Consult Clin Psychol 2001;69:942-9.

53 Grossman P, Kappos L, Gensicke H, et al. MS quality of life, depression, and fatigue improve after mindfulness training: a randomized trial. Neurology 2010:75:1141-9.

54 Stuifbergen AK, Becker $\mathrm{H}$, Perez $\mathrm{F}$, et al. A randomized controlled trial of a cognitive rehabilitation intervention for persons with multiple sclerosis. Clin Rehabil 2012:26:882-93.

55 Artemiadis AK, Vervainioti AA, Alexopoulos EC, et al. Stress management and multiple sclerosis: a randomized controlled trial. Arch Clin Neuropsychol 2012;27:406-16.

56 Nazari F, Soheili M, Hosseini S, et al. A comparison of the effects of reflexology and relaxation on pain in women with multiple sclerosis. J Complement Integr Med 2016;13:65-71.

57 Hughes CM, Smyth S, Lowe-Strong AS. Reflexology for the treatment of pain in people with multiple sclerosis: a double-blind randomised sham-controlled clinical trial. Mult Scler 2009:15:1329-38

58 Bastani F, Sobhani M, Emamzadeh Ghasemi HS. Effect of acupressure on fatigue in women with multiple sclerosis. Glob J Health Sci 2015;7:375-81.

59 Morrow SA, Rosehart $\mathrm{H}$. Effects of single dose mixed amphetamine salts--extended release on processing speed in multiple sclerosis: a double blind placebo controlled study. Psychopharmacology 2015;232:4253-9.

60 James E, Dobson R, Kuhle J, et al. The effect of vitamin D-related interventions on multiple sclerosis relapses: a meta-analysis. Mult Scler 2013;19:1571-9.

61 Hempel S, Graham GD, Fu N, et al. A systematic review of the effects of modifiable risk factor interventions on the progression of multiple sclerosis. Mult Scler 2017;23:513-24.

62 National Institute for Health and Care Excellence (NICE). Multiple sclerosis: management of multiple sclerosis in primary and secondary care. National Clinical Guideline Centre 2014;186.

63 Yadav V, Bever C, Bowen J, et al. Summary of evidence-based guideline: complementary and alternative medicine in multiple sclerosis: report of the guideline development subcommittee of the American Academy of Neurology. Neurology 2014;82:1083-92. 